\title{
ON THE IDENTITY OF TWO $q$-DISCRETE PAINLEVÉ EQUATIONS AND THEIR GEOMETRICAL DERIVATION
}

\author{
B. GRAMMATICOS, A. RAMANI, AND T. TAKENAWA
}

Received 9 October 2005; Revised 5 January 2006; Accepted 5 January 2006

We show that two recently discovered $q$-discrete Painleve equations are one and the same system. Moreover we provide a novel derivation of this $q$-discrete system based on transformations obtained with the help of affine Weyl groups.

Copyright (c) 2006 B. Grammaticos et al. This is an open access article distributed under the Creative Commons Attribution License, which permits unrestricted use, distribution, and reproduction in any medium, provided the original work is properly cited.

\section{Introduction}

One of the characteristics of discrete Painlevé equations is that they may possess more than one canonical form. Indeed we often encounter equations which are written as a system involving several dependent variables. Since by definition the discrete Painlevé equations are second-order mappings, these multicomponent systems include equations which are local. It is then straightforward, if some equation is linear in one of the variables, to solve for this variable and eliminate it from the final system. One thus obtains two perfectly equivalent forms which may have totally different aspects.

This feature is in contrast with the continuous Painlevé case where the latitude left by the transformations which preserve the Painleve property is minimal. The fact that there exist just 6 continuous Painlevé equations at second order while the number of possible second-order discrete Painlevé equations is in principle infinite may play a role. The possible existence of an unlimited number of discrete Painlevé equations has been explicitly pointed out in [2]. In that paper we have, in fact, presented a novel definition for the discrete Painlevé equations. The traditional definition of a discrete Painlevé equation is that of an integrable, nonautonomous, second-order mapping, the continuous limit of which is a continuous Painlevé equation. This definition turned out to be severely limitative since it binds the discrete systems to the continuous ones through the continuous limit. However, as was shown repeatedly, the discrete systems are more fundamental than their continuous counterparts, and in the case of discrete Painlevé equations much richer, as far as the degrees of freedom are concerned. We were thus naturally led in [2] to propose

Hindawi Publishing Corporation Advances in Difference Equations Volume 2006, Article ID 36397, Pages 1-11

DOI 10.1155/ADE/2006/36397 


\section{On the identity of two $q$-discrete Painlevé equations}

a novel definition of discrete Painlevé equations, which is now a discrete system defined by a periodic repetition of a given nonclosed pattern on the weight lattice of the affine Weyl group $\mathrm{E}_{8}^{(1)}$ or one of its degenerations.

This proliferation of discrete Painlevé equations raises the question of the independence of the various forms. Indeed, while one can, in principle, construct an unlimited number of such systems, there exists no a priori guarantee that they are all different. This is something to be assessed for each case at hand. In this paper we will concentrate on two recently discovered discrete Painlevé equations and show that they are one and the same equation. Moreover we will provide a novel derivation of this $q$-discrete system based on the geometrical approach we have developed in [10].

\section{The two discrete Painlevé systems}

In a recent paper, Kajiwara et al. [6] have introduced the following system:

$$
\begin{aligned}
& \bar{f}_{0}=a_{0} a_{1} f_{1} \frac{1+a_{2} f_{2}+a_{2} a_{3} f_{2} f_{3}+a_{2} a_{3} a_{0} f_{2} f_{3} f_{0}}{1+a_{0} f_{0}+a_{0} a_{1} f_{0} f_{1}+a_{0} a_{1} a_{2} f_{0} f_{1} f_{2}}, \\
& \bar{f}_{1}=a_{1} a_{2} f_{2} \frac{1+a_{3} f_{3}+a_{3} a_{0} f_{3} f_{0}+a_{3} a_{0} a_{1} f_{3} f_{0} f_{1}}{1+a_{1} f_{1}+a_{1} a_{2} f_{1} f_{2}+a_{1} a_{2} a_{3} f_{1} f_{2} f_{3}}, \\
& \bar{f}_{2}=a_{2} a_{3} f_{3} \frac{1+a_{0} f_{0}+a_{0} a_{1} f_{0} f_{1}+a_{0} a_{1} a_{2} f_{0} f_{1} f_{2}}{1+a_{2} f_{2}+a_{2} a_{3} f_{2} f_{3}+a_{2} a_{3} a_{0} f_{2} f_{3} f_{0}}, \\
& \bar{f}_{3}=a_{3} a_{0} f_{0} \frac{1+a_{1} f_{1}+a_{1} a_{2} f_{1} f_{2}+a_{1} a_{2} a_{3} f_{1} f_{2} f_{3}}{1+a_{3} f_{3}+a_{3} a_{0} f_{3} f_{0}+a_{3} a_{0} a_{1} f_{3} f_{0} f_{1}},
\end{aligned}
$$

with

$$
a_{0} a_{1} a_{2} a_{3}=\lambda,
$$

and the "bar" indicates the evolution along the independent discrete variable. The latter was introduced by taking

$$
f_{0} f_{2}=f_{1} f_{3}=\gamma z
$$

whereupon one finds that $z$ is of the form $\lambda^{n}$ and thus the system is a $q$-discrete equation. The inverse evolution of $(2.1)$ is given by

$$
\begin{aligned}
& \underline{f}_{0}=\frac{f_{3}}{a_{0} a_{1}} \frac{a_{2} a_{1} a_{0}+a_{1} a_{0} f_{2}+a_{0} f_{2} f_{1}+f_{2} f_{1} f_{0}}{a_{0} a_{3} a_{2}+a_{3} a_{2} f_{0}+a_{2} f_{0} f_{3}+f_{0} f_{2} f_{3}}, \\
& \underline{f}_{1}=\frac{f_{0}}{a_{1} a_{2}} \frac{a_{3} a_{2} a_{1}+a_{2} a_{1} f_{3}+a_{1} f_{3} f_{2}+f_{3} f_{2} f_{1}}{a_{1} a_{0} a_{3}+a_{0} a_{3} f_{1}+a_{3} f_{1} f_{0}+f_{1} f_{0} f_{3}}, \\
& \underline{f}_{2}=\frac{f_{1}}{a_{2} a_{3}} \frac{a_{0} a_{3} a_{2}+a_{3} a_{2} f_{0}+a_{2} f_{0} f_{3}+f_{0} f_{2} f_{3}}{a_{2} a_{1} a_{0}+a_{1} a_{0} f_{2}+a_{0} f_{2} f_{1}+f_{2} f_{1} f_{0}}, \\
& \underline{f}_{3}=\frac{f_{0}}{a_{3} a_{0}} \frac{a_{1} a_{0} a_{3}+a_{0} a_{3} f_{1}+a_{3} f_{1} f_{0}+f_{1} f_{0} f_{3}}{a_{3} a_{2} a_{1}+a_{2} a_{1} f_{3}+a_{1} f_{3} f_{2}+f_{3} f_{2} f_{1}} .
\end{aligned}
$$


This system was studied in detail by Masuda [7] who has shown that its continuous limit is the Painlevé $\mathrm{V}$ equation.

From (2.1) we find that the dependent variables satisfy the relations

$$
\bar{f}_{0} \bar{f}_{2}=\lambda f_{1} f_{3}, \quad \bar{f}_{1} \bar{f}_{3}=\lambda f_{0} f_{2} .
$$

However the constraint $f_{0} f_{2}=f_{1} f_{3}$ is unwarranted. One can perfectly relinquish it and obtain a valid discrete Painlevé equation. Thus it is possible to assume

$$
f_{0} f_{2}=\gamma z, \quad f_{1} f_{3}=\delta z, \quad \bar{f}_{0} \bar{f}_{2}=\delta \bar{z}, \quad \bar{f}_{1} \bar{f}_{3}=\gamma \bar{z},
$$

where $\bar{z}=\lambda z$, whereupon the equation acquires one more degree of freedom. This extension was introduced by one of us (T. Takenawa) in [13] where it was shown that the geometry of the evolution of this extended equation, together with its Schlesinger transformations, can be described by the affine Weyl group $\mathrm{D}_{5}^{(1)}$. By using the freedom of the origin of $z$, we can define $z=\sqrt{\gamma \delta} \lambda^{n}$ and find, finally,

$$
f_{0} f_{2}=k z, \quad f_{1} f_{3}=\frac{z}{k},
$$

where $k=\sqrt{\gamma / \delta}$. Of course from $(2.5)$ we find that $\bar{f}_{0} \bar{f}_{2}=\bar{z} / k$ and similarly $\bar{f}_{1} \bar{f}_{3}=k \bar{z}$.

In another recent paper [11] two of the present authors (A. Ramani and B. Grammaticos), in collaboration with Willox et al., have examined the limits of the $q-\mathrm{P}_{\mathrm{VI}}$ equation [1]

$$
\frac{\left(x_{n} x_{n+1}-z_{n} z_{n+1}\right)}{\left(x_{n} x_{n-1}-z_{n} z_{n-1}\right)} \frac{\left(x_{n} x_{n+1}-1\right)}{\left(x_{n} x_{n-1}-1\right)}=\frac{\left(x_{n}-a z_{n}\right)\left(x_{n}-z_{n} / a\right)\left(x_{n}-b z_{n}\right)\left(x_{n}-z_{n} / b\right)}{\left(x_{n}-c\right)\left(x_{n}-1 / c\right)\left(x_{n}-d\right)\left(x_{n}-1 / d\right)},
$$

where $z_{n}=z_{0} \lambda^{n}$ and $a, b, c, d$ are the parameters of the equation. By letting $a \rightarrow \infty$ and $c \rightarrow \infty$ simultaneously, we found the equation

$$
\frac{\left(x_{n} x_{n+1}-z_{n} z_{n+1}\right)\left(x_{n} x_{n-1}-z_{n} z_{n-1}\right)}{\left(x_{n} x_{n+1}-1\right)\left(x_{n} x_{n-1}-1\right)}=f z_{n} \frac{\left(x_{n}-b z_{n}\right)\left(x_{n}-z_{n} / b\right)}{\left(x_{n}-d\right)\left(x_{n}-1 / d\right)},
$$

where $f$ stands for the ratio $a / c$. As we have shown the equation has $\mathrm{P}_{\mathrm{V}}$ as a continuous limit. Again, the form (2.9) does not encapsulate the full freedom of the equation and an extension is possible. This can be obtained either by starting from (2.9) and extending it with the help of some discrete integrability criterion [3], [4] or by starting from the "asymmetric" form of (2.8) which incorporates the maximal number of parameters. To make a long story short the extended form of (2.9) turns out to be

$$
\frac{\left(x_{n} x_{n+1}-z_{n} z_{n+1}\right)\left(x_{n} x_{n-1}-z_{n} z_{n-1}\right)}{\left(x_{n} x_{n+1}-1\right)\left(x_{n} x_{n-1}-1\right)}=\frac{f z_{n}}{\theta_{n}} \frac{\left(x_{n}-\theta_{n} b z_{n}\right)\left(x_{n}-\theta_{n} z_{n} / b\right)}{\left(x_{n}-d\right)\left(x_{n}-1 / d\right)}
$$

with $\log \theta_{n}=\alpha(-1)^{n}$. As we have pointed out in [11], the geometry of the transformations of this equation is related to the affine Weyl group $\mathrm{D}_{5}^{(1)}$, just as in the case of (2.1). 
This result is not a coincidence. As we will show, the two equations are identical. In order to show this we introduce the variables $x \equiv f_{0}, y \equiv f_{1}$ and use the relations $f_{2}=$ $k z / x, f_{3}=z /(k y)$. The evolution equations for $x$ can now be written as

$$
\begin{aligned}
& x_{n} x_{n+1}=\frac{z_{n} z_{n+1}\left(a_{0} x_{n}+1\right)+y_{n} k z_{n+1} / a_{3}+a_{0} a_{1} x_{n} y_{n}}{1+a_{0} x_{n}+k y_{n} z_{n+1} / a_{3}+a_{0} a_{1} x_{n} y_{n}}, \\
& x_{n} x_{n-1}=\frac{k y_{n} z_{n} z_{n-1}\left(x+a_{0}\right)+x_{n} z_{n} / a_{3}+k z_{n} z_{n-1} a_{0} a_{1}}{k y_{n}\left(x_{n}+a_{0}\right)+x_{n} z_{n} / a_{3}+k z_{n} z_{n-1} a_{0} a_{1}} .
\end{aligned}
$$

Next we eliminate $y$ between the two equations and reorganise the result. We find

$$
\frac{\left(x_{n} x_{n+1}-z_{n} z_{n+1}\right)\left(x_{n} x_{n-1}-z_{n} z_{n-1}\right)}{\left(x_{n} x_{n+1}-1\right)\left(x_{n} x_{n-1}-1\right)}=\frac{a_{1} z_{n}}{k a_{3}} \frac{\left(x_{n}+k z_{n} a_{2}\right)\left(x_{n}+k z_{n} / a_{2}\right)}{\left(x_{n}+a_{0}\right)\left(x_{n}+1 / a_{0}\right)}
$$

which is exactly (2.10) with $\theta_{n}=k$. This specific choice is due to the fact that we have written the equation around $x_{n}$. Had we written the equation around $x_{n \pm 1}$, we would have found (2.10) with $\theta_{n \pm 1}=1 / k$. Thus (2.1) is perfectly equivalent to (2.10).

\section{A derivation using discrete Miura transformations}

In [6] the derivation of (2.1) was based on the analysis of discrete dynamical systems associated to extended affine Weyl groups of type $A_{m}^{(1)} \times A_{n}^{(1)}$. The derivation of (2.7), on the other hand, as mentioned above, was based on the limits of equations related to the $\mathrm{E}_{7}^{(1)}$ affine Weyl group. However as explained in [11, 13], (2.1) and (2.7) can be connected to the affine Weyl group $\mathrm{D}_{5}^{(1)}$. It is thus natural to present a derivation of these systems (and here we choose (2.9) for simplicity reasons) based on the Miura transformations obtained from the geometry of $\mathrm{D}_{5}^{(1)}$.

In [10] we have studied in detail the geometry of the "asymmetric" $q-\mathrm{P}_{\mathrm{III}}$ [9], which was shown by Jimbo and Sakai [5] to be a discrete form of $\mathrm{P}_{\mathrm{VI}}$, and we have found that it is described by the affine Weyl group $\mathrm{D}_{5}^{(1)}$. This equation was the first for which the property of self-duality was established. What we mean by self-duality is that the same equation describes the evolution along the independent variable or among any of the parameters of the equation (the latter evolution being mediated by the Schlesinger transformations). In this sense all the parameters, including the independent variable, play the same role. The form of the "asymmetric" $q-\mathrm{P}_{\mathrm{III}}$ we are going to use in what follows is

$$
\begin{gathered}
\hat{y} y=\frac{(x+a p / q)(x+1 /(a p q))}{(1+x a /(p q))(1+x p /(a q))} \\
x \hat{x}=\frac{(\hat{y}+r / \hat{q})(\hat{y}+1 /(r \hat{q}))}{(1+\hat{y} /(s \hat{q}))(1+s \hat{y} / \hat{q})},
\end{gathered}
$$

where the "hat" symbol is used in order to indicate evolution along the $q$ direction, that is, $\hat{q}=\lambda q$. The form of (3.1) is chosen so as to indicate that the $x$ variable exists only on "even" lattice sites while the $y$ variable exists only on "odd" sites with respect to the evolution of $q$. 
Next we consider an evolution along the $p$ variable and use the "tilde" symbol for it, that is, $\tilde{p}=\lambda p$, while in the derivation that follows the parameters $a, r$, and $s$ remain constant. A new dependent variable $w$ is introduced through the Miura transformations

$$
\begin{gathered}
\underset{w}{\hat{y}}=\frac{a x+1 /(p q)}{1+a x /(p q)}=y \tilde{\wedge}, \\
\hat{y} \hat{\hat{w}}=\frac{a \widehat{\hat{x}}+1 /(p \widehat{\hat{q}})}{1+a \hat{\hat{x}} /(p \hat{\hat{q}})} .
\end{gathered}
$$

We now solve (3.2) and (3.3) for $x$ and $\hat{x}$ and use (3.1b) in order to obtain an equation involving just $\hat{y}, \underset{\sim}{\mathcal{w}}$, and $\hat{\widetilde{w}}$ :

$$
\left(\frac{\underset{w}{w} \hat{y}-1 /(p q)}{\underset{\sim}{w} \hat{y} /(p q)-1}\right)\left(\frac{\hat{y} \hat{\tilde{w}}-1 /(p \hat{\hat{q}})}{\hat{y} \hat{\tilde{w}} /(p \hat{\hat{q}})-1}\right)=\frac{1}{a^{2}} \frac{(\hat{y}+r / \hat{q})(\hat{y}+1 /(r \hat{q}))}{(1+\hat{y} /(s \hat{q}))(1+s \hat{y} / \hat{q})}
$$

In order to bring (3.4) under canonical form we introduce formally $Y=y / q$ and $W=$ $w / p$. By this generic notation we mean that one has to use the local value of $p$ or $q$. We remind at this point that $p$ is invariant under the "hat" evolution and similarly $q$ does not change when we follow the "tilde" evolution. We find thus

$$
\left(\frac{\underset{\sim}{W} \hat{Y}-1 /\left(p^{2} q^{2}\right)}{\underset{\sim}{W}-1}\right)\left(\frac{\hat{Y}-\hat{\tilde{W}}-1 /\left(p^{2} \hat{\tilde{q}}^{2}\right)}{\hat{\hat{Y}} \hat{W}-1}\right)=\frac{1}{a^{2} p^{2}} \frac{\left(\hat{Y}+r / \hat{q}^{2}\right)\left(\hat{Y}+1 /\left(r \hat{q}^{2}\right)\right)}{(1+\hat{Y} / s)(1+s \hat{Y})} .
$$

As can be assessed by inspection, (3.5) describes an evolution along an "oblique" direction where a single step is a combination of two steps, one in each of the "hat" and "tilde" directions. We are thus led into introducing formally the new independent variable

$$
Z=\frac{1}{p q}
$$

whereupon the quantity $1 /\left(p^{2} q^{2}\right)$ on the left-hand side of (3.5) can be consistently rewritten as $\hat{Z} \underset{\sim}{Z}$. Similarly we have $1 /\left(p^{2} \hat{\hat{q}}^{2}\right)=\hat{Z} \hat{\tilde{Z}}$. Moreover introducing the auxiliary quantity $g=p / \hat{q}$ we can give finally (3.5) into a form which, with the appropriate interpretation, is identical to (2.12):

$$
\left(\frac{\underset{\sim}{W} \hat{Y}-\hat{Z} Z}{\underset{\sim}{W} \hat{Y}-1}\right)\left(\frac{\hat{Y} \hat{\tilde{W}}-\hat{Z} \hat{\tilde{Z}}}{\hat{\hat{Y}} \hat{W}-1}\right)=\frac{\hat{Z}}{a^{2} g} \frac{(\hat{Y}+r g \hat{Z})(\hat{Y}+g \hat{Z} / r)}{(1+\hat{Y} / s)(1+s \hat{Y})} .
$$

We proceed now, along similar lines, to derive the second equation of the system. First we 
6 On the identity of two $q$-discrete Painlevé equations

write the dual equations of (3.1):

$$
\begin{aligned}
& \tilde{w} \underset{\sim}{w}=\frac{(x+1 /(a p q))(x+a q / p)}{(1+x a /(p q))(1+q x /(a p))}, \\
& \underset{\approx}{\approx}=\frac{(\underset{\sim}{\mathcal{w}}+r / \underset{\sim}{p})(\underset{\sim}{w}+1 /(r \underset{\sim}{p}))}{(1+s \underset{\sim}{w} / \underset{\sim}{p})(1+\underset{\sim}{w} /(s p))} .
\end{aligned}
$$

Next we solve for $x$ from the leftmost equality of (3.2) and downshifting the rightmost equality of (3.2) twice along the tilde direction we solve it for $\underset{\approx}{x}$. Using (3.8b) we can now obtain an equation involving just $\underset{\sim}{w}, \hat{y}$, and $\underset{\approx}{y}$. We find

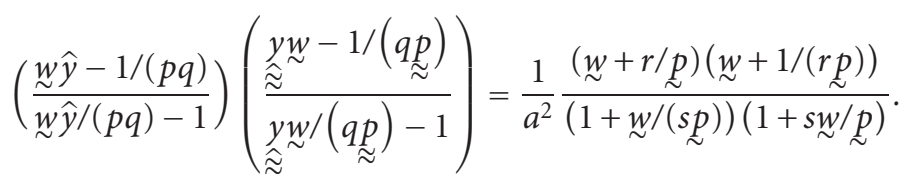

Again in order to bring the equation to canonical form we use the variables $Y$ and $W$. Without entering into all the tedious but straightforward manipulations, we give the form of the final equation

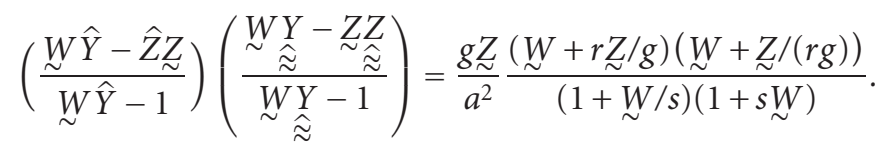

This equation complements (3.7). We must point out here that the parameter $g$ has shifted position, with respect to (3.7), from numerator to denominator and vice versa, in perfect agreement with $(2.10)$, where $\log \theta_{n}=\alpha(-1)^{n}$.

\section{Relation to the Weyl group}

In this section, we present explicit relations of these discrete Painlevé equations to the extended Weyl group of type $\mathrm{D}_{5}^{(1)}$, and discuss their space of initial conditions in the spirit of the Okamoto-Sakai approach $[8,12]$. Let us define the transformations $w_{i}(i=$ $0,1, \ldots, 5), \sigma_{01}$, and $\pi$ on the space $(x, y ; a, p, q, r, s, \lambda) \in \mathbb{C}^{2} \times$ (the parameter space) as follows: $w_{0}$ maps $(x, y ; a, p, q, r, s, \lambda)$ to

$$
\left(x, y ; \frac{1}{p}, \frac{1}{a}, q, r, s, \lambda\right)
$$

$w_{1}$ maps it to

$$
(x, y ; p, a, q, r, s, \lambda)
$$

$w_{2}$ maps it to

$$
\left(x, \frac{a y(x+p q / a)}{q(x+a p / q)} ; q, p, a, r, s, \lambda\right)
$$


$w_{3}$ maps it to

$$
\left(\frac{\lambda \sqrt{r / s} x(y+q s / \lambda)}{q(y+r \lambda / q)}, y ; a, p, \lambda \sqrt{\frac{r}{s}}, \frac{q \sqrt{r s}}{\lambda}, \frac{\lambda \sqrt{r s}}{q}, \lambda\right),
$$

$w_{4}$ maps it to

$$
\left(x, y ; a, p, q, \frac{1}{r}, s, \lambda\right)
$$

$w_{5}$ maps it to

$$
\left(x, y ; a, p, q, r, \frac{1}{s}, \lambda\right)
$$

$\sigma_{01}$ maps it to

$$
\left(x, \frac{1}{y} ; \frac{1}{a}, p, \frac{1}{q}, \frac{1}{r}, \frac{1}{s}, \frac{1}{\lambda}\right),
$$

$\pi$ maps it to

$$
\left(y, x ; \sqrt{\frac{r}{s}}, \sqrt{r s}, \frac{q}{\lambda}, a p, \frac{p}{a}, \frac{1}{\lambda}\right) .
$$

From Sakai's theory on the relation to rational surfaces, the transformations we introduced here act on the root basis $\left(\alpha_{0}, \alpha_{1}, \alpha_{2}, \alpha_{3}, \alpha_{4}, \alpha_{5}\right)$ as

$$
w_{i}\left(\alpha_{j}\right)=\alpha_{j}+\left(\alpha_{i}, \alpha_{j}\right) \alpha_{i},
$$

where the bilinear form $\left(\alpha_{i}, \alpha_{j}\right)$ is given by the Cartan matrix of negative sign of type $\mathrm{D}_{5}^{(1)}$;

$$
\begin{aligned}
\sigma_{01}:\left(\alpha_{0}, \alpha_{1}, \alpha_{2}, \alpha_{3}, \alpha_{4}, \alpha_{5}\right) & \longmapsto\left(\alpha_{1}, \alpha_{0}, \alpha_{2}, \alpha_{3}, \alpha_{4}, \alpha_{5}\right), \\
\pi:\left(\alpha_{0}, \alpha_{1}, \alpha_{2}, \alpha_{3}, \alpha_{4}, \alpha_{5}\right) & \longmapsto\left(\alpha_{4}, \alpha_{5}, \alpha_{3}, \alpha_{2}, \alpha_{0}, \alpha_{1}\right) .
\end{aligned}
$$

They generate the extended affine Weyl group of type $\mathrm{D}_{5}^{(1)}$.

Below we give the list of mappings and their actions on the parameter space and on the root basis. The dependent variables that appear below correspond to the following diagram:

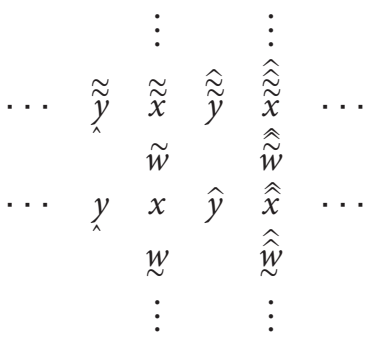


8 On the identity of two $q$-discrete Painlevé equations

(i) The map $w(3.1 \mathrm{a}):(x, y) \mapsto(x, \hat{y})$ defined by (3.1a) is described by the generators as

$$
\begin{gathered}
w(3.1 \mathrm{a})=w_{5} \circ w_{4} \circ w_{1} \circ w_{0} \circ w_{2} \circ w_{1} \circ w_{0} \circ \sigma_{01} \circ w_{2}: \\
(x, y ; a, p, q, r, s, \lambda) \longmapsto\left(x, \frac{(x+a p / q)(x+1 /(a p q))}{y(1+x a /(p q))(1+x p /(a q))} ; a, p, q, r, s, \frac{1}{\lambda}\right), \\
\vec{\alpha} \longmapsto\left(-\alpha_{0},-\alpha_{1},-\alpha_{2}, \alpha_{0}+\alpha_{1}+2 \alpha_{2}+\alpha_{3}+\alpha_{4}+\alpha_{5},-\alpha_{4},-\alpha_{5}\right)
\end{gathered}
$$

(1) $w(3.1 \mathrm{~b}):(x, \hat{y}) \mapsto(\hat{x}, \hat{y})$,

$$
\begin{gathered}
w(3.1 \mathrm{~b})=w_{0} \circ w_{1} \circ w_{4} \circ w_{5} \circ w_{3} \circ w_{4} \circ w_{5} \circ \pi \circ \sigma_{01} \circ \pi \circ w_{3}: \\
\left(x, y ; a, p, q, r, s, \frac{1}{\lambda}\right) \longmapsto\left(\frac{(\hat{y}+r /(q \lambda))(\hat{y}+1 /(q r \lambda))}{x(1+\hat{y} /(q s \lambda))(1+\hat{y} s /(q \lambda))}, \hat{y} ; a, p, q \lambda^{2}, r, s, \lambda\right), \\
\vec{\alpha} \longmapsto\left(-\alpha_{0},-\alpha_{1}, \alpha_{0}+\alpha_{1}+\alpha_{2}+2 \alpha_{3}+\alpha_{4}+\alpha_{5},-\alpha_{3},-\alpha_{4},-\alpha_{5}\right) .
\end{gathered}
$$

(ii) Miura transformation $w(3.2):(x, \hat{y}) \mapsto(x, \underset{\sim}{w})$,

$$
\begin{gathered}
w(3.2)=w_{5} \circ w_{4} \circ \sigma_{01} \circ w_{0} \circ w_{1} \circ w_{2} \circ w_{0}: \\
(x, \hat{y} ; a, p, q, r, s, 1 / \lambda) \longmapsto\left(x, \frac{a x+1 /(p q)}{\hat{y}(1+a x /(p q))} ; q, a, p, r, s, \lambda\right), \\
\vec{\alpha} \longmapsto\left(-\alpha_{0}-\alpha_{2}, \alpha_{1}+\alpha_{2},-\alpha_{1}, \alpha_{0}+\alpha_{1}+\alpha_{2}+\alpha_{3}+\alpha_{4}+\alpha_{5},-\alpha_{4},-\alpha_{5}\right) .
\end{gathered}
$$

(iii) The other Miura $w(3.3):(\hat{\hat{x}}, \hat{y}) \mapsto(\hat{\hat{x}}, \widehat{\hat{\tilde{w}}})$ is the same as $w(3.2)$,

$$
\begin{gathered}
w(3.3)=w_{5} \circ w_{4} \circ \sigma_{01} \circ w_{0} \circ w_{1} \circ w_{2} \circ w_{0}: \\
\left(\hat{x}, \hat{y} ; a, p, q \lambda^{2}, r, s, \lambda\right) \longmapsto\left(\hat{\hat{x}}, \frac{a \hat{x}+1 /\left(p q \lambda^{2}\right)}{\hat{y}\left(1+a \hat{x} /\left(p q \lambda^{2}\right)\right)} ; q \lambda^{2}, a, p, r, s, \lambda\right) .
\end{gathered}
$$

The action on the root basis is the same as that of $w(3.2)$.

(2) $w(3.8 \mathrm{a}):(x, \underset{\sim}{w}) \mapsto(x, \tilde{w})$ is the same as $w(3.1 \mathrm{a})$,

$$
w_{5} \circ w_{4} \circ w_{1} \circ w_{0} \circ w_{2} \circ w_{1} \circ w_{0} \circ \sigma_{01} \circ w_{2}:
$$

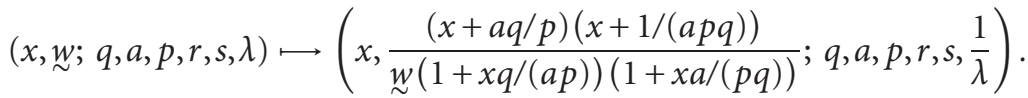

The action on the root basis is the same as that of $w$ (3.1a).

(3) $w(3.8 \mathrm{~b}): \underset{\sim}{\underset{\sim}{x}}, \underset{\sim}{\mathcal{w}}) \mapsto(x, \underset{\sim}{\mathcal{w}})$ is the same as $w(3.1 \mathrm{~b})$,

$w_{0} \circ w_{1} \circ w_{4} \circ w_{5} \circ w_{3} \circ w_{4} \circ w_{5} \circ \pi \circ \sigma_{01} \circ \pi \circ w_{3}:$

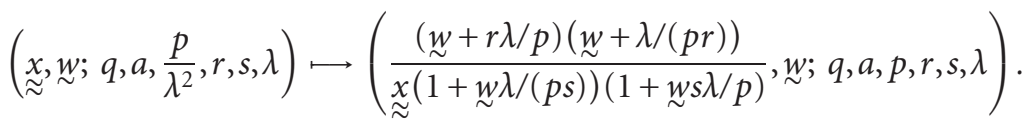

The action on the root basis is the same as that of $w(3.1 \mathrm{~b})$. 
(4) $w(3.1 \mathrm{~b}) \circ w(3.1 \mathrm{a})=w(3.8 \mathrm{~b}) \circ w(3.8 \mathrm{a})$ acts on the root basis as

$$
\vec{\alpha} \longmapsto\left(\alpha_{0}, \alpha_{1}, \alpha_{2}-\delta, \alpha_{3}+\delta, \alpha_{4}, \alpha_{5}\right),
$$

where $\delta=\alpha_{0}+\alpha_{1}+2 \alpha_{2}+2 \alpha_{3}+\alpha_{4}+\alpha_{5}$ is the null vector orthogonal to any basis; thus this mapping is a translation of the Weyl group.

(iv) The mapping $w(3.2)^{-1} \circ w(3.8 \mathrm{~b}) \circ w(3.8 \mathrm{a}) \circ w(3.2):(x, \hat{y}) \mapsto(\tilde{\tilde{x}}, \hat{\tilde{y}})$ acts on the root basis as

$$
\vec{\alpha} \longmapsto\left(\alpha_{0}+\delta, \alpha_{1}-\delta, \alpha_{2}, \alpha_{3}, \alpha_{4}, \alpha_{5}\right),
$$

thus, this sequence defines a translation of the Weyl group in another direction.

Next, we consider the "diagonal mappings" $w(3.7):(\hat{y}, \underset{w}{w}) \mapsto(\hat{y}, \widehat{\hat{\tilde{w}}})$ and $w(3.10)$ : $((\underset{\approx}{\sim}, \underset{\sim}{w}) \mapsto(\hat{y}, \underset{\sim}{w}))$ from the Weyl group theoretical point of view. However, these mappings do not belong to the same representation of the Weyl group. The above mappings $w$ (3.1a), and so forth can be lifted to the automorphism of a family of rational surfaces, which are obtained from $\mathbf{p}^{1}(\mathbb{C}) \times \mathbf{p}(\mathbb{C}) \ni(x, y)$ by 2 times blowing-up on each line $x=0$, $x=\infty, y=0$, or $y=\infty$. These rational surfaces are called "space of initial conditions" in the sense of Okamoto-Sakai $[8,12]$. For example, $w(3.1 \mathrm{a})$ can be lifted to an isomorphism from a rational surface obtained by blowups at

$$
\begin{gathered}
(x, y)=\left(-\frac{a p}{q}, 0\right),\left(-\frac{1}{a p q}, 0\right),\left(-\frac{p q}{a}, \infty\right),\left(-\frac{a q}{p}, \infty\right), \\
\left(0,-\frac{r \lambda}{q}\right),\left(0,-\frac{\lambda}{q r}\right),\left(\infty,-\frac{s q}{\lambda}\right),\left(0,-\frac{q}{s \lambda}\right)
\end{gathered}
$$

to a rational surface obtained by blowups at

$$
\begin{gathered}
(x, \hat{y})=\left(-\frac{a p}{q}, 0\right),\left(-\frac{1}{a p q}, 0\right),\left(-\frac{p q}{a}, \infty\right),\left(-\frac{a q}{p}, \infty\right), \\
\left(0,-\frac{r}{q \lambda}\right),\left(0,-\frac{1}{q r \lambda}\right),(\infty,-q s \lambda),\left(0,-\frac{q \lambda}{s}\right) .
\end{gathered}
$$

All elements of the above Weyl group preserve these parametrization, but the space of initial conditions for $w$ (3.7) and $w$ (3.10) has different parametrization. Actually, $w(3.7)$ : $(\hat{y}, \underset{\sim}{w}) \mapsto(\hat{y}, \hat{\widetilde{w}})$ is lifted to the isomorphism from a rational surface obtained by blowups at

$$
\begin{gathered}
(\hat{y}, \underset{\sim}{w})=(\infty, 0),(0, \infty),\left(-\frac{\lambda}{q r},-\frac{r \lambda}{p}\right),\left(-\frac{r}{q \lambda},-\frac{\lambda}{p r}\right),\left(-\frac{q \lambda}{s},-\frac{p s}{\lambda}\right),\left(-q s \lambda,-\frac{p}{s \lambda}\right), \\
\left(\frac{1}{\hat{y}}, \underset{\sim}{\underset{\sim}{w}}\right)=\left(0, \frac{p\left(1-a^{2} q^{2}\right)}{q\left(p^{2}-a^{2}\right)}\right), \quad\left(\hat{y}, \frac{1}{\hat{y} \underset{\sim}{w}}\right)=\left(0, \frac{p\left(q^{2}-a^{2}\right)}{q\left(1-a^{2} p^{2}\right)}\right)
\end{gathered}
$$


to a rational surface obtained by blowups at

$$
\begin{aligned}
(\hat{y}, \hat{\tilde{w}}) & =(\infty, 0),(0, \infty),\left(-\frac{r}{q \lambda},-\frac{1}{p r \lambda}\right),\left(-\frac{1}{q r \lambda},-\frac{r}{p \lambda}\right),\left(-q s \lambda,-\frac{p \lambda}{s}\right),\left(-\frac{q \lambda}{s},-p s \lambda\right), \\
\left(\frac{1}{\hat{y}}, \hat{y} \hat{\tilde{w}}\right) & =\left(0, \frac{p\left(1-a^{2} q^{2} \lambda^{4}\right)}{q \lambda^{2}\left(p^{2}-a^{2}\right)}\right), \quad\left(\hat{y}, \frac{1}{\hat{y} \hat{\tilde{w}}}\right)=\left(0, \frac{p\left(q^{2} \lambda^{4}-a^{2}\right)}{q \lambda^{2}\left(1-a^{2} p^{2}\right)}\right) .
\end{aligned}
$$

These two different parametrizations are connected by Miura transformations, for example, a mapping $w_{d}(3.2):(\hat{y}, \underset{\sim}{w}) \mapsto(x, \hat{y})$, which is not an element of the Weyl group. In order to avoid these complications, it is sufficient to consider parallelograms instead of diagonal lines, that is, for example, the mapping $w(3.2)^{-1} \circ w(3.8 \mathrm{~b}) \circ w(3.3) \circ w(3.1 \mathrm{~b})$ :

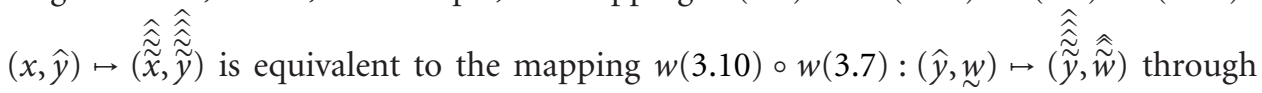
Miura transformations $w_{d}(3.2)$, and so forth and it acts on the root basis as

$$
\vec{\alpha} \longmapsto\left(\alpha_{0}+\delta, \alpha_{1}-\delta, \alpha_{2}+\delta, \alpha_{3}-\delta, \alpha_{4}, \alpha_{5}\right)
$$

This difference can be explained at the level of the diagram (4.11) by the fact that only vertically or horizontally adjoined pairs of dependent variables are mapped to each other by our $\mathrm{D}_{5}^{(1)}$ Weyl group.

\section{Conclusions}

In this paper we have examined two different $q$-discrete Painlevé equations. The first one was derived by Kajiwara et al. [6] in the form of a system of four depenent variables subject to two constraints. Under this form, the equation was shown by Masuda [7] to be a $q$-discrete analogue of $\mathrm{P}_{\mathrm{V}}$. The constraints were shown [13] to be too restrictive and the equation was extended accordingly. The second system was obtained [11] from a special limit of $q-\mathrm{P}_{\mathrm{VI}}$. In the present paper we have shown that, despite their radically different forms, the two systems are in fact the same mapping. The geometry of both equations (since it was not known that they coincided) was given in $[11,13]$ as being that of the affine Weyl group $\mathrm{D}_{5}^{(1)}$, but no explicit construction was offered. In the present paper we have provided the missing link and have explicitly derived the equation at hand from the elementary Miura transformations of $\mathrm{D}_{5}^{(1)}$. Further, we have clarified these relations from the point of view of Weyl group theory and of rational surfaces.

\section{References}

[1] B. Grammaticos and A. Ramani, On a novel q-discrete analogue of the Painlevé VI equation, Physics Letters. A 257 (1999), no. 5-6, 288-292.

[2] __ Generating discrete Painlevé equations from affine Weyl groups, Regular \& Chaotic Dynamics 10 (2005), no. 2, 145-152.

[3] B. Grammaticos, A. Ramani, and V. Papageorgiou, Do integrable mappings have the Painlevé property?, Physical Review Letters 67 (1991), no. 14, 1825-1828. 
[4] J. Hietarinta and C. Viallet, Singularity confinement and chaos in discrete systems, Physical Review Letters 81 (1998), no. 2, 325-328.

[5] M. Jimbo and H. Sakai, A q-analog of the sixth Painlevé equation, Letters in Mathematical Physics 38 (1996), no. 2, 145-154.

[6] K. Kajiwara, M. Noumi, and Y. Yamada, Discrete dynamical systems with $W\left(A_{m-1}^{(1)} \times A_{n-1}^{(1)}\right)$ symmetry, Letters in Mathematical Physics 60 (2002), no. 3, 211-219.

[7] T. Masuda, On the rational solutions of q-Painlevé Vequation, Nagoya Mathematical Journal 169 (2003), 119-143.

[8] K. Okamoto, Sur les feuilletages associés aux équations du second ordre à points critiques fixes de $P$. Painlevé, Japanese Journal of Mathematics. New Series 5 (1979), no. 1, 1-79.

[9] G. R. W. Quispel, J. A. G. Roberts, and C. J. Thompson, Integrable mappings and soliton equations. II, Physica D. Nonlinear Phenomena 34 (1989), no. 1-2, 183-192.

[10] A. Ramani, Y. Ohta, J. Satsuma, and B. Grammaticos, Self-duality and Schlesinger chains for the asymmetric $\mathrm{d}-\mathrm{P}_{\mathrm{II}}$ and $\mathrm{q}-\mathrm{P}_{\mathrm{III}}$ equations, Communications in Mathematical Physics 192 (1998), no. $1,67-76$.

[11] A. Ramani, R. Willox, B. Grammaticos, A. S. Carstea, and J. Satsuma, Limits and degeneracies of discrete Painlevé equations: a sequel, Physica A: Statistical and Theoretical Physics 347 (2005), no. $1-4,1-16$.

[12] H. Sakai, Rational surfaces associated with affine root systems and geometry of the Painleve equations, Communications in Mathematical Physics 220 (2001), no. 1, 165-229.

[13] T. Takenawa, Weyl group symmetry of type $D_{5}^{(1)}$ in the q-Painlevé V equation, Funkcialaj Ekvacioj. Serio Internacia 46 (2003), no. 1, 173-186.

B. Grammaticos: GMPIB, Université Paris VII, Tour 24-14, 5e étage, 75251 Paris, France

E-mail address: grammati@paris7.jussieu.fr

A. Ramani: CPT, Ecole Polytechnique, CNRS, UMR 7644, 91128 Palaiseau, France

E-mail address: ramani@cpht.polytechnique.fr

T. Takenawa: Faculty of Marine Technology, Tokyo University of Marine Science and Technology,

2-1-6 Etchu-jima, Koto-ku, 135-8533 Tokyo, Japan

E-mail address: takenawa@e.kaiyodai.ac.jp 